\title{
Using Behavioral Skills Training to Teach Peer Models: Effects on Interactive Play for Students with Moderate to Severe Disabilities
}

\author{
Alyssa Covey • Tangchen $\mathrm{Li} \cdot$ Sheila R. Alber-Morgan
}

Accepted: 11 December 2020/Published online: 7 January 2021

(C) Association for Behavior Analysis International 2021

\begin{abstract}
This study examined the effects of using behavioral skills training (BST) to teach peer models to engage students with moderate to severe developmental disabilities in interactive play. Two separate multiplebaseline across participants designs were used to determine the effectiveness of BST on the peer models' implementation of the procedural steps and the target students' percent of intervals engaged in interactive play. Results demonstrated that BST was functionally related to the peer models' accurate implementation of procedures and the target students' percentage of intervals engaged in interactive play. In addition, all participants demonstrated generalization to novel activities and play partners, and three of the four target students maintained high levels of interactive play for up to 13 weeks after intervention.
\end{abstract}

Keywords Behavior skills training · Peer mediated instruction · Interactive play · Developmental disabilities

Playing with friends is a vital part of childhood that all children, including those with disabilities, should be

This manuscript is based on Alyssa Covey's master's thesis.

\author{
A. Covey \\ Worthington City Schools, Columbus, OH, USA \\ T. Li $\cdot$ S. R. Alber-Morgan $(\bowtie)$ \\ The Ohio State University, A356 PAES Building, 305 Annie and \\ John Glen Avenue, Columbus, OH 43229, USA \\ e-mail: morgan.651@osu.edu
}

able to enjoy. Research demonstrates a range of benefits for engaging in play, including increased independent participation in a range of settings (Frey \& Kaiser, 2011) and increased social and communication skills (Lifter, Foster-Sanda, Arzamarski, Briesch, \& McClure, 2011). However, many children with moderate to severe developmental disabilities experience persistent difficulty engaging in interactive play activities with peers (e.g., Harrison \& Oakland, 2015; Kemp, 2016; Simpson \& Bui, 2016). Difficulty with cooperative play activities can reduce opportunities for children to practice important social, language, and cognitive skills in natural contexts as well as hinder their ability to build and maintain friendships (Bredecamp, 2019; Cook, Klein, \& Chen, 2020; Dennis \& Stockall, 2015; Patry \& Horn, 2020).

Fortunately, children with a range of disabilities can acquire, maintain, and generalize appropriate social play behaviors through explicit instruction. In their review of play interventions, Jung and Sainato (2013) identified video and live modeling, systematic prompting strategies (e.g., least-to-most prompting), and pivotal response training (i.e., naturalistic teaching) among effective interventions for teaching play. Most of the studies examined intervention packages consisting of explicit instruction (i.e., verbal instructions, modeling, prompting, and reinforcement) delivered by adults. Research also demonstrates that directly teaching peers to model, prompt, and reinforce play behaviors of children with disabilities increases their social engagement and language production (e.g., Barton, Choi, \& Mauldin, 2019; Katz \& Girolametto, 2013; Zhang \& Wheeler, 
2011). For example, Milam, Hemmeter, and Barton (2020) taught preschool peer buddies the "Stay, Play, Talk" strategy to socially engage preschoolers with ASD during play time. The interventionist used modeling, role-playing, and prompting (visual and verbal) to teach peer buddies to stay close to their friend, bring a toy, initiate interactive play, and talk about the toy they are playing with (e.g., "The car goes fast."). Results demonstrated increases in social interactions for all participants as well as maintenance of the strategy after fading adult supports. Peer-mediated interventions (PMI) have a long history of empirical support demonstrating improved social, academic, and functional skills for students with a range of disabilities (e.g., Dart, Collins, Klingbeil, \& McKinley, 2014; Lee, Odom, \& Loftin, 2007; Maheady, Sacca, \& Harper, 1987; Strain, Shores, \& Timm, 1977).

PMI interventions require effective training of peers so they can implement the interventions consistently. behavior skills training (BST) is an evidence-based practice for training individuals to acquire, maintain, and generalize their learning across a variety of skills and populations (e.g., Clayton \& Headley, 2019; Drifke, Tiger, \& Wierzba, 2016; Fetherston \& Sturmey, 2014; Kornacki, Ringdahl, Sjostrom, \& Nuernberger, 2013; Sawyer et al., 2017). BST consists of four components: instruction, modeling, rehearsal, and feedback. During instruction, participants learn the importance of the target skill, its components, and how and when to implement it (Ervin, Wilson, Maynard, \& Bramblett, 2018; Singh et al., 2017). During modeling, participants are shown how to perform the desired behaviors or skills through live demonstration or video modeling (Morgan \& Wine, 2018; Singh et al., 2017). The rehearsal and feedback components of BST occur in conjunction with one another. As participants rehearse the skill through role-playing, the trainer provides immediate, specific, and corrective feedback and praise about the participant's performance (Singh et al., 2017).

Although BST is an evidence-based intervention for training parents, teachers, and paraprofessionals, few studies have examined the effects of using BST to train peers to deliver interventions to students with disabilities. In one study, Tarasenko, Miltenberger, Brower-Breitwieser, and Bosch (2010) used BST to train participants to implement abduction prevention skills with their younger peers.
Peer models implemented all four components of BST (instructions, modeling, rehearsal, and feedback). Results demonstrated that peer trainers successfully used BST to teach their younger peers abduction prevention strategies to mastery.

In another BST study with peers as interventionists, Ervin et al. (2018) trained peer models to appropriately respond to target students' disruptive behaviors. The BST intervention consisted of a discussion about the reason for the disruptive behaviors and the importance of responding appropriately; modeling the appropriate responses; roleplaying; and providing corrective feedback. Results demonstrated that BST was effective for increasing the appropriate responses of peer models to their classmates' disruptive behaviors. Although not directly targeted, this study also demonstrated an overall decrease in disruptive classroom behavior. Using a similar BST training procedure, Mathews, Vatland, Lugo, Koenig, and Gilroy (2018) demonstrated quick skill acquisition by peer models on initiating verbal interaction, prompting of skills, and delivering praise. Additional contingencies, such as posting data and providing prompts were required to achieve generalization of prompting and praise behaviors.

Research on using BST to train peer models has been implemented with peers aged 7 to 18 across different skill domains (i.e., social skills, academic skills, safety skills). BST intervention research demonstrates successful outcomes across a range of learners. However, previous research has not examined the effects of using BST to train peer models to implement play interventions with students who have moderate to severe disabilities. The purpose of this study was to examine the effects of BST on peer models' correct implementation of a play intervention and target students' engagement in play. In particular, this study was designed to answer the following research questions.

1. What are the effects of BST on the acquisition, maintenance, and generalization of procedural steps implemented correctly by peer models?

2. What are the effects of using BST to train peer models on the percentage of intervals engaged in interactive play by students who have moderate to severe disabilities?

3. What are the peer models' opinions of the BST intervention? 


\section{Method}

\section{Participants}

This study was conducted in a public school in the Midwest region of the United States. There were eight participants: four peer models without disabilities and four target students with moderate to severe disabilities. All four peer models (Abby, Bethany, Megan, and Nicole) were sixth-grade girls who were 12 years old at the time of the study. The peer models were selected as participants based on their previous participation as peer models in the multiple-disabilities classroom that was a regular practice in place at the school. Table 1 shows demographic and school-related information for the peer models.

The target students with disabilities were three boys and a girl (Kevin, Caleb, Quinn, and Rachel) ranging from 6 to 8 years old and diagnosed with a range of disabilities including ASD, intellectual disability, cerebral palsy, and MPS III. The teacher recruited target participants based on their social skills deficits and their IEP goals targeting interactive play skill development. Table 2 shows demographic and school related information for the target students.

The peer models and target students were randomly paired prior beginning baseline data collection. These pairings remained consistent throughout the experiment. Tables 1 and 2 show which peer models were paired with which target students.

\section{Setting}

All data were collected in a self-contained special education classroom for children with multiple disabilities. During experimental sessions, this classroom consisted of one teacher, two assistants, six to eight students with

Table 1 Peer models information

\begin{tabular}{lllll}
\hline & $\begin{array}{l}\text { Nicole } \\
\text { (with } \\
\text { Kevin) }\end{array}$ & $\begin{array}{l}\text { Bethany } \\
\text { (with Caleb) }\end{array}$ & $\begin{array}{l}\text { Abby (with } \\
\text { Quinn) }\end{array}$ & $\begin{array}{l}\text { Megan (with } \\
\text { Rachel) }\end{array}$ \\
\hline Gender & Female & Female & Female & Female \\
Age & 12 & 12 & 12 & 12 \\
Grade & 6 th & 6 th & 6 th & 6th \\
Ethnicity & Caucasian & Caucasian & Caucasian & African \\
& & & & American \\
\hline
\end{tabular}

disabilities, one to four peer models, and sometimes an additional data collector. The classroom teacher, who was a student in a master's degree program at the time of the study, was the experimenter, primary data collector, and first author. A doctoral student served as a second observer who collected IOA and treatment integrity data.

\section{Recruiting and Informed Consent Process}

After receiving IRB approval, packets that included a recruiting letter explaining the study and a permission form to sign were sent to the parents of the target students and the parents of the peer models. After receiving signed parent permission forms, the special education teacher (i.e., experimenter) obtained assent from the peer models by reading an assent script to each peer model individually and privately. The script included an explanation of the procedures, risks, and benefits, and the option to withdraw at any time without penalty. If the peer model agreed to participate, she provided verbal assent and signed the assent form. Due to their cognitive level of functioning, the IRB did not require assent from the target students who were all diagnosed with moderate to severe disabilities.

\section{Definition and Measurement of Dependent Variables}

This study examined the effects of the BST intervention on the percentage of procedural steps implemented correctly by peer models and percentage of intervals engaged in interactive play by target students with disabilities. Data were collected two to four times per week and once per day depending on the participants' schedules.

Percentage of Procedural Steps Implemented Correctly by Peer Models This dependent variable assessed the peer models' implementation of the procedural steps for each play activity. The teacher created a 16-step task analysis listing the procedural steps and used it to record the peer model's implementation of each step (see Appendix 1). A plus sign (+) was recorded for each step performed independently and a minus sign (-) was recorded if the step was skipped or required prompting. Depending on the target students' responses to prompting, some steps did not apply during every session in which case an N/A was recorded for that step. Percentage of procedural steps was calculated by dividing the number of steps completed independently by the 
Table 2 Students with moderate to severe disabilities information

\begin{tabular}{|c|c|c|c|c|}
\hline & $\begin{array}{l}\text { Kevin (with } \\
\text { Nicole) }\end{array}$ & Caleb (with Bethany) & Quinn (with Abby) & Rachel (with Megan) \\
\hline Gender & Male & Male & Male & Female \\
\hline Age & 8 & 8 & 6 & 6 \\
\hline Grade & 2 nd & 2 nd & Kindergarten & Kindergarten \\
\hline Ethnicity & Caucasian & Caucasian & $\begin{array}{l}\text { Multiracial (Caucasian and } \\
\text { African American) }\end{array}$ & Caucasian \\
\hline Diagnosis & ASD & Intellectual Disability and ADHD & $\begin{array}{l}\text { Cerebral Palsy and Multiple } \\
\text { Disabilities }\end{array}$ & ASD; MPS III \\
\hline $\begin{array}{l}\text { Communication } \\
\text { Style }\end{array}$ & $\begin{array}{l}\text { Nonverbal; LAMP } \\
\text { app on iPad }\end{array}$ & $\begin{array}{l}\text { Partially verbal; vocalizations } \\
\text { plus LAMP app on iPad }\end{array}$ & Nonverbal; accent device & $\begin{array}{l}\text { Partially verbal; vocalizations } \\
\text { plus picture symbols }\end{array}$ \\
\hline $\begin{array}{l}\text { Time in Gen. } \\
\text { Ed. }\end{array}$ & $2 \mathrm{~h} 8 \mathrm{~min}$ & $2 \mathrm{~h} 8 \mathrm{~min}$ & $1 \mathrm{~h} 45 \mathrm{~min}$ & $1 \mathrm{~h} 45 \mathrm{~min}$ \\
\hline
\end{tabular}

total number of steps (not including those marked N/A) and multiplying by 100 .

\section{Percentage of Intervals Engaged in Interactive} Play Interactive play, defined as joint interaction between the peer model and the target student, was demonstrated when the target student was taking turns with a peer engaging in the following activities: rolling a ball back and forth, building a structure of blocks, and racing toy cars. Target students were considered not engaged in play if they played alone (away from the peer), played next to the peer with no interaction, or moved away from the play area. Data collection for each 5-min session began after the teacher said, "It is playtime."

Partial interval recording was used to measure the percentage of intervals engaged in play. In particular, data were collected during 10-s intervals over a 5-min observation period. The total duration of play time was 5 $\min$. The special education teacher used a MotivAider ${ }^{\circledR}$ timer set at 10-s intervals and recorded a plus sign (+) if interaction occurred at any time during the interval, or a minus sign (-) if interaction did not occur at any time during the interval. Percentage of intervals engaged in interactive play was calculated by dividing the number of intervals marked with a plus sign by the total number of intervals and multiplying by 100 .

\section{Interobserver Agreement (IOA)}

IOA data were collected to determine agreement on the percentage of steps in the task analysis completed accurately by peer models and the percentage of intervals engaged in interactive play by the target students. For peer models, item-by-item IOA data were collected to determine agreement on steps performed correctly. IOA was calculated by dividing the number of agreements by the number of agreements plus disagreements and multiplying by 100 . IOA data were collected for both dependent variables on $45 \%$ of the experimental sessions across all phases of the study. For target students' interactive play, IOA was calculated by dividing the number of intervals where agreement occurred by the total number of intervals and multiplying by 100 .

Table 3 shows IOA for each participant across experimental phases. IOA for time engaged in interactive play across target students and phases ranged between $80 \%$ and $100 \%$ with a mean of $93 \%$. The IOA for number of BST steps implemented correctly by peer models ranged between $81 \%$ and $100 \%$ with a mean of $95 \%$.

\section{Treatment Integrity}

Treatment integrity was assessed on $21 \%$ of the experimental sessions to determine the extent to which the teacher (i.e., the experimenter) followed the experimental procedures in each phase of the study as planned. An observer used a checklist and recorded whether or not the teacher completed each step of the experimental procedure. Treatment integrity ranged from $80 \%$ to $100 \%$ with a mean of $98 \%$ across phases.

\section{Social Validity}

At the end of the study, the teacher administered an experimenter-designed social validity questionnaire to 
Table 3 Interobserver agreement for target students and peer models

\begin{tabular}{|c|c|c|c|c|c|c|c|c|}
\hline & \multicolumn{4}{|c|}{ Target students } & \multicolumn{4}{|l|}{ Peer models } \\
\hline & Kevin & Quin & Caleb & Rachel & Nicole & Megan & Abby & Bethany \\
\hline$\%$ of IOA & $14 \%$ & $40 \%$ & $38 \%$ & $21 \%$ & $14 \%$ & $28 \%$ & $45 \%$ & $40 \%$ \\
\hline Range & $80 \%-100 \%$ & $80 \%-96 \%$ & $83 \%-100 \%$ & $87 \%-100 \%$ & $81 \%-100 \%$ & $87 \%-100 \%$ & $93 \% 100 \%$ & $87 \%-100 \%$ \\
\hline Mean & $91 \%$ & $90 \%$ & $96 \%$ & $95 \%$ & $92 \%$ & $95 \%$ & $99 \%$ & $94 \%$ \\
\hline
\end{tabular}

the peer models to assess their acceptability of the intervention. The questionnaire contained five statements (I liked being taught how to implement play activities through instruction, modeling, and roleplaying while getting feedback from the teacher; I liked being able to practice more if I needed to; I liked having the task analysis to help me remind myself of the steps; I liked participating in this study; and I feel that I am better able to play with and help my peers with disabilities than I was before this study) for which the peer model responded by circling the word "agree," "disagree," or "neutral." The peer models completed their questionnaires independently in different locations to prevent influencing one another's responses.

\section{Experimental Design}

This study used a multiple baseline across participants design to examine the effects of BST training on the number of intervals engaged in interactive play by the target students and the number of BST steps completed accurately by the peer models. The experimental conditions were baseline, intervention (Phase 1 and 2), generalization, and maintenance.

\section{Procedures}

Baseline Baseline data were collected on the percentage of procedural steps peer models completed and the duration of interactive play between peer models and target students. The special education teacher set out play materials for each of three activities (rolling the ball, building blocks, and racing cars) on alternating baseline sessions. The experimenter observed each pair for 5-min sessions beginning after delivery of the cue "It is play time." No instructions were provided to either of the participants peers nor the target students. The peer model with the most stable baseline (or descending trend) began the intervention phase first. Each subsequent peer model began intervention after the previous participant achieved at least $80 \%$ of the procedural steps on three consecutive sessions.

Intervention Phase 1: BST with Peer Models The special education teacher conducted BST sessions individually with each of the peer models during a 20-min time block in the afternoon. She provided the peer models with a 16-step task analysis for each of three interactive play activities: rolling ball to each other, stacking blocks, and racing cars. Each task analysis consisted of inviting the target student to play, using least-to-most prompting for each step in the play activity (i.e., verbal prompt, picture prompt, hand-over-hand prompt), and praising the target student for interactive play behaviors. The special education teacher provided instructions using the task analysis, modeled the task analysis steps, role-played the steps with the peer model, and provided corrective feedback and praise.

Intervention Phase 2: Interactive Play Activity Peer models began implementing the procedural steps for one of the three interactive play activities after the special education teacher cued "It is playtime." During the activities, the peer models had access to the task analysis for reference. Interactive play sessions were $5 \mathrm{~min}$ long. The play activities (rolling the ball, building blocks, and racing cars) were alternated randomly on each intervention session. On some sessions, the experimenter selected the activity. On other sessions, either the target student or the peer model chose the activity. This selection varied across participants and the experimenters did not record which sessions they were allowed to choose or which participant selected the activity.

Maintenance During the maintenance phase, the task analyses were not provided to the peer models and the procedures were the same as in baseline. The 
maintenance phase began 5 to 10 weeks after the last intervention session and lasted up to 13 weeks. The time between the first maintenance probe and the subsequent maintenance probes varied due to the participants' availability and scheduling. Three maintenance probes were completed for each pair of participants.

Generalization Using the same procedures as in intervention, probes were administered during 5-min observation sessions throughout the intervention phase to determine if the target students and peer models could generalize their play behaviors to different peer models and to untrained play activities. Generalization probes sometimes occurred on the same day as intervention sessions and sometimes the following day. These activities aligned with the procedural checklist steps for the three trained activities and included the following games: Poppa's Pizza Topple, Legos, Jenga, and Spill the Beans. At least three generalization probes were administered to the peer models and target students.

\section{Results}

Peer Models

Figure 1 shows the percentage of BST steps implemented correctly by the peer models. All four peer models showed considerable variability during baseline with steps completed accurately ranging from 5\% to $100 \%$. Bethany and Nicole had the lowest average percentage of steps completed accurately with $23 \%$ and $26 \%$, respectively. Abby and Megan had the highest average with $46 \%$ and $47 \%$, respectively. During intervention, each peer model demonstrated an immediate and substantial increase of steps completed accurately, ranging from $80 \%$ to $100 \%$ across participants. After baseline, data for all four peer models remained stable at $80 \%$ to $100 \%$ throughout the intervention, maintenance, and generalization conditions.

\section{Target Students}

Figure 2 shows the percentage of intervals engaged in interactive play by target students. During intervention and maintenance, all four students demonstrated increased intervals of interactive play above baseline level. Each target student's baseline data showed differing levels of variability. Percentage of engaged intervals ranged between $0 \%$ and $100 \%$, with 36 of the 38 total baseline data points at less than 50\%. Caleb's and Rachel's baseline data, which ranged between $0 \%$ and $25 \%$ intervals engaged, were more stable than Quinn's and Kevin's whose data showed a few outlying data points that overlapped with the intervention data.

During intervention, all four target students showed an immediate increase of engaged intervals with Quinn and Kevin showing the greatest increase. Caleb increased percentage of engaged intervals from an average of $6 \%$ in baseline to an average of $62.25 \%$ in intervention, $73.33 \%$ in maintenance, and $61.75 \%$ during generalization. Quinn showed an increase in engaged intervals from an average of $14.44 \%$ in baseline to an average of $77.57 \%$ in the intervention, and $81.33 \%$ in maintenance. During intervention, Quinn's data show a downward trend prior to a 1-month absence. A generalization probe conducted for Quinn during maintenance shows $70 \%$ engaged intervals. Rachel increased the percentage of intervals engaged in interactive play from an average of $8.5 \%$ in baseline to an average of $42.63 \%$ in the intervention, $29 \%$ in maintenance, and $36.66 \%$ in generalization. Kevin showed an increase in the percentage of intervals engaged from an average of $17.62 \%$ in baseline to an average of $80.11 \%$ in intervention, $87.66 \%$ in maintenance, and $77.66 \%$ in generalization.

\section{Social Validity}

All four peer models indicated their opinions of the BST intervention by circling agree on $60 \%-100 \%$ of questions. The lowest rating by the peer models was neutral. On three out of four questionnaires completed by the peer models, the statement "I liked having the task analysis to help me remind myself of the steps" was rated neutral. Nicole also responded neutral to "I liked being able to practice more if I needed to." Bethany responded agree to all five statements on the questionnaire. All peer models responded agree to the statement "I feel that I am better able to play with and help my peers with disabilities than I was before this study."

\section{Discussion}

The findings of this study demonstrate that BST training was functionally related to the correct implementation of procedural steps by peer models and resulted in increased intervals engaged in interactive play by students 
Fig. 1 Percentage of steps

implemented correctly by peer models

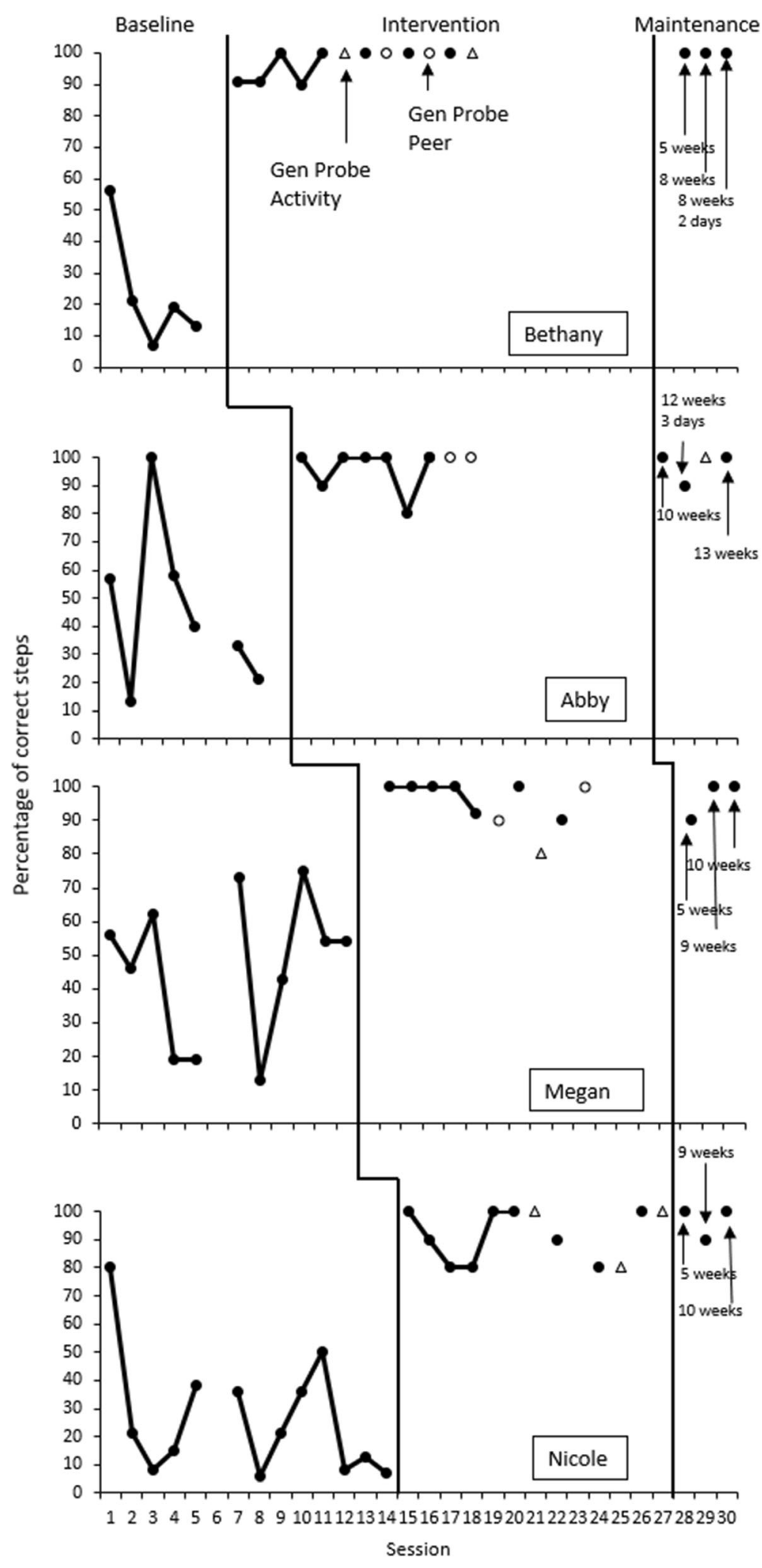


Fig. 2 Percentage of interactive play of target students

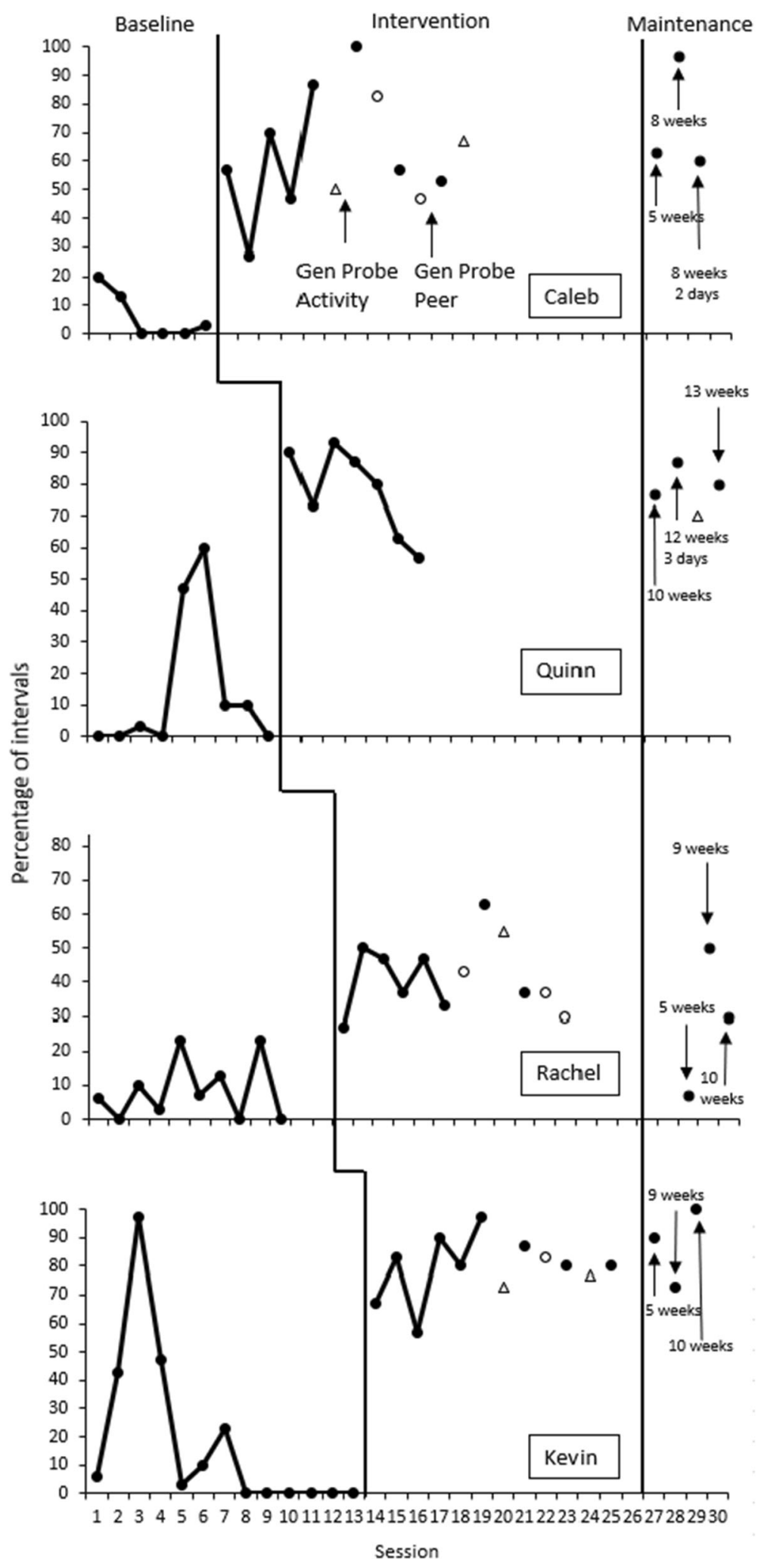


with moderate to severe disabilities. The peer models also demonstrated generalization to other activities and play partners as well as maintenance for up to 13 weeks after intervention. These results are consistent with the positive outcomes of previous BST research (e.g., Clayton \& Headley, 2019; Drifke et al., 2016; Fetherston \& Sturmey, 2014; Sawyer et al., 2017) and extends those findings to typically developing peer models who provided a peer-mediated intervention to students with moderate to severe disabilities. In addition to improved performance, results of the social validity questionnaires showed peer models' overall satisfaction of the BST procedures.

Consistent with previous research, BST was effective for producing quick and significant improvements of peer models' implementation of procedural steps that were durable over 2 to 3 months. Most important, this intervention resulted in increased engagement of interactive play for the target students with disabilities which generalized to novel play activities and play partners. Students with disabilities are most likely to benefit from interactive play with peers when they can stay engaged with them. An important implication of this study is that a teacher of students with moderate to severe disabilities can implement a systematic procedure that is effective for increasing interactive play with peers.

\section{Limitations and Future Research}

Limitations of this study were related to time constraints and scheduling, the age gap between the peer models and target students, the way the peer models and target students were paired, the type of play activities selected (and who selected them), and the timing of the generalization probes. This experiment was conducted in a public school, so data collection was confined to the time constraints of the school calendar as well as the established schedules of the peer models and target students. In addition, Quinn (a target student) was absent for several consecutive sessions during the intervention phase so we were unable to obtain generalization data for him. Although enough data were collected to demonstrate a functional relation, future research may be enhanced by additional experimental sessions, especially those that assess generalization.

Another limitation is that, due to minor classroom disruptions on two sessions, the teacher only collected data for one of the two students in the pair (i.e., the target student). In particular, on Session 6, the teacher did not collect data for the two peer models (Bethany and Abby) and on Session 9, she did not collect data for Abby. She only collected data for the target students on those sessions. This type of limitation may be inherent in research in which a practitioner's responsibility for ongoing classroom instruction and management may interfere with observation sessions. Future research may address this limitation by using videotaped sessions or additional observers.

Considering that there are downward trends for the target students during intervention, it might be surprising that some of the target students' intervals of interactive play were higher in maintenance than during intervention. A possible explanation for this is the target students may have had additional opportunities to practice play outside of the classroom and were possibly more receptive to interacting with peers.

Regarding the assessment of generalization, another limitation is that no generalization probes were taken during the baseline phase. Although the participants' apparent generalized outcomes are promising, we were unable to determine if generalization was functionally related to the BST intervention because there was no comparison generalization data in baseline. Also, the generalization measure was limited to a different trained peer model and a different activity. In addition to collecting generalization data during baseline, future research should attempt to expand the generalization measures to other settings or other play partners.

Another limitation was the 4- to 6-year age gap between the peer models and target students. Prior to implementing this study, the 12-year-old peer models volunteered in the special education classroom as part of their regular school schedule. The researchers did not have access to younger students as peer models. Future research should examine the extent to which the intervention would be effective for peer models and target students closer in age. Another possible limitation regarding pair selection is that peer models and target students were matched randomly rather than strategically. Strategic pairing by student preferences, skill levels, or personalities may produce better results than random pairing. Future research should examine various ways to pair target students with peer models to determine which would produce optimum results.

The types of interactive play activities selected (i.e., rolling a ball, stacking blocks, and racing cars) was another possible limitation. To reduce the 
probability of satiation with a particular activity, three different activities were alternated throughout the study. When using multiple activities to measure interactive play, the activities should be of approximately equal effort, interest, and ease for participants. Based on the spikes in baseline (which occurred during the ball-rolling activity), this was not the case in this present study. Future research should attempt to experimentally identify activities that are similar enough to produce stable responding across activities. In addition, the experimenter sometimes provided choices for activity type, and sometimes she did not. Date were not collected on whether the session was a choice or a nonchoice session. It is possible that the participants (either peer model or target student) performed better when they selected the activity. Future research should investigate the effects of choice on BST training and interactive play.

Future research should also explore ways to increase the efficiency of training peer models as well as identifying peer model behaviors that result in the best outcomes for the target students. For example, the BST task analysis for training peer models could be adjusted so that it works for multiple target students, regardless of their behavioral or motor needs. For example, the task analysis can be simplified for students who struggle with the series of directions. Future researchers should also consider teaching peer models to use specific praise and to vary praise statements during interactive play sessions. Manipulating the variable of praise may increase target students' motivation to continue to engage in interactive play. Future research should also investigate the effects of BST with peer models on target students' improvement with other social skills, such as engaging in reciprocal conversations, giving compliments, or asking for help.

Implications for Practitioners

A particular strength of this study is that a practitioner (i.e., the special education teacher) implemented the intervention with her students and the peer models thereby increasing the likelihood continuing an effective intervention, which she is planning to do. Unfortunately, due to Covid-19, the teacher was unable to continue using the strategy with her students. The teacher who implemented this study was a master's degree student in an applied behavior analysis program so she may not be representative of many special education teachers. Other teachers may require more training to accomplish effective peer-mediated interventions. Considering that play is important for the development of social and communication skills, language skills, problemsolving skills, and cognitive skills, practitioners should make efforts to facilitate and encourage interactive play with peers.

Teachers should consider various ways to use BST within peer-mediated interventions in their classrooms. For example, teachers can train peer models in groups to increase the efficiency of training. Teachers can also provide direct instruction to target students and equip them with the skills needed to respond to peer models' initiation appropriately. Students with disabilities can also be directly taught to initiate play with peers. To promote generalization of peer-mediated interventions, teachers should provide frequent opportunities for practice and systematically vary aspects of the instructional environment such as the peer models, types of activities and materials, time of day, classroom or community setting, and types of prompts (e.g., verbal, visual, physical) and reinforcers (social, tangible) used during instruction and interactive play.

\section{Compliance with Ethical Standards}

Conflicts of Interest The authors report no conflicts of interest related to this research manuscript.

Research Involving Human Participants and/or Animals The experimenters received approval from the university's institutional review board before recruiting participants and beginning the study.

Informed Consent Prior to beginning data collection, the experimenters obtained parents' permission for the participants to participate in the study using an IRB approved informed consent process. See "Recruiting and Informed Consent Process".

\section{Appendix 1}

Task Analysis

1. Peer student invites target student to play.

2. Peer student shows target student the toy. 
3. Peer student sits down and prompts target student to sit the verbal cue "sit down" paired with a picture symbol.

4. Peer student makes first interactive play attempt (e.g., rolling the ball, stacking a block, pushing a car) with target student.

5. Peer student waits for target student to reciprocate the interactive play (e.g., rolling the ball, stacking a block, pushing a car).

6. If target student reciprocates play, peer student praises the target student (e.g., "I like how you , great playing!") paired with a high five or fist bump.

7. If target student does not reciprocate play, peer student prompts target student to reciprocate the play by using verbal cues paired with picture symbols.

8. If the target student does not reciprocate play after the verbal and visual cues, peer student uses hand over hand to prompt response from target student (e.g., rolling the ball, stacking a block, pushing a car).

9. If target students leaves the area, peer student cues the target student to come play using least to most prompting (verbal, visual, physical).

10. Peer student makes another interactive play attempt (e.g., rolling the ball, stacking a block, pushing a car).

11. Peer student waits for target student to reciprocate the interactive play (e.g., rolling the ball, stacking a block, pushing a car)

12. If target student reciprocates play, peer student praises the target student saying, "I like how you , great playing!" paired with a high-five or fist bump.

13. If target student does not reciprocate play, peer student prompts target student to reciprocate the play by using verbal cues paired with picture symbols.

14. If the target student does not reciprocate play after the verbal and visual cues, peer student uses physical prompting (e.g., rolling the ball, stacking a block, pushing a car).

15. If target student leaves the area, peer student cues for the target student to come play using least to most prompting.

16. Peer student makes another interactive play attempt (e.g., rolling the ball, stacking a block, pushing a car).

\section{References}

Barton, E. E., Choi, G., \& Mauldin, E. G. (2019). Teaching sequences of pretend play to children with disabilities. Journal of Early Intervention, 41(1), 13-29. https://doi. org/10.1177/1053815118799466.

Bredecamp, S. (2019). Effective practices in early childhood education: Building a foundation (3rd ed.). Hoboken, New Jersey: Pearson.

Clayton, M., \& Headley, A. (2019). The use of behavioral skills training to improve staff performance of discrete trial training. Behavioral Interventions, 34(1), 136-143. https://doi. org/10.1002/bin.1656.

Cook, R. E., Klein, M. D., \& Chen, D. (2020). Adapting early childhood curricula for children with disabilities and special needs (10th ed.). Hoboken, New Jersey: Pearson.

Dart, E. H., Collins, T. A., Klingbeil, D. A., \& McKinley, L. E. (2014). Peer management interventions: A meta-analytic review of single-case research. School Psychology Review, 43(4), 367-384. https://doi.org/10.17105/SPR-14-0009.1.

Dennis, L. R., \& Stockall, N. (2015). Using play to build the social competence of young children with language delays: Practical guidelines for teachers. Early Childhood Education Journal, 43(1), 1-7. https://doi.org/10.1007 /s10643-014-0638-5.

Drifke, M. A., Tiger, J. H., \& Wierzba, B. C. (2016). Using behavioral skills training to teach parents to implement three-step prompting: A component analysis and generalization assessment. Learning \& Motivation, 57(2), 1-14. https://doi.org/10.1016/j.lmot.2016.12.001.

Ervin, T., Wilson, A. N., Maynard, B. R., \& Bramblett, T. (2018). Determining the effectiveness of behavior skills training and observational learning on classroom behaviors: A case study. Social Work Research, 42(2), 106-117. https://doi. org/10.1093/swr/svy005.

Fetherston, A. M., \& Sturmey, P. (2014). The effects of behavioral skills training on instructor and learner behavior across responses and skill sets. Research in Developmental Disabilities, 35(2), 541-562. https://doi.org/10.1016/j. ridd.2013.11.006.

Frey, J. R., \& Kaiser, A. P. (2011). The use of play expansions to increase the diversity and complexity of object play in young children with disabilities. Topics in Early Childhood Special Education, 31, 99-111. https://doi.org/10.1177 /0271121410378758.

Harrison, P., \& Oakland, T. (2015). Adaptive behavior assessment system [Measurement instrument]. Retrieved from https://www.wpspublish.com/store/p/3234/adaptivebehavior-assessment-system-third-edition-abas-3

Jung, S., \& Sainato, D. M. (2013). Teaching play skills to young children with autism. Journal of Intellectual \& Developmental Disability, 38(1), 74-90. https://doi. org/10.3109/13668250.2012.732220.

Katz, E., \& Girolametto, L. (2013). Peer-mediated intervention for preschoolers with ASD implemented in early childhood education settings. Topics in Early Childhood Special Education, 33(3), 133-143. https://doi.org/10.1177 /0271121413484972.

Kemp, K. (2016). Teaching social skills to students with autism spectrum disorders and students with intellectual disabilities 
(Unpublished doctoral dissertation). New York, NY: Columbia University. https://doi.org/10.7916/D8R78D99.

Kornacki, L. T., Ringdahl, J. E., Sjostrom, A., \& Nuernberger, J. E. (2013). A component analysis of a behavioral skills training package used to teach conversation skills to young adults with autism spectrum and other developmental disorders. Research in Autism Spectrum Disorders, 7(11), 1370-1376. https://doi.org/10.1016/j.rasd.2013.07.012.

Lee, S., Odom, S. L., \& Loftin, R. (2007). Social engagement with peers and stereotypic behavior of children with autism. Journal of Positive Behavior Interventions, 9(2), 67-79. https://doi.org/10.1177/10983007070090020401.

Lifter, K., Foster-Sanda, S., Arzamarski, C., Briesch, J., \& McClure, E. (2011). Overview of play: Its uses and importance in early intervention/early childhood special education. Infants \& Young Children, 24(3), 225-245. https://doi. org/10.1097/IYC.0b013e31821e995c.

Maheady, L., Sacca, M. K., \& Harper, G. F. (1987). Classwide student tutoring teams: The effects of peer-mediated instruction on the academic performance of secondary mainstreamed students. Journal of Special Education, 21(3), 107-121. https://doi.org/10.1177 /002246698702100309.

Mathews, T. L., Vatland, C., Lugo, A. M., Koenig, E. A., \& Gilroy, S. P. (2018). Training peer models to promote social skills: Considerations for practice. Focus on Autism \& Other Developmental Disabilities, 33(3), 160-170. https://doi. org/10.1177/1088357617735814.

Milam, M. E., Hemmeter, M. L., \& Barton, E. E. (2020). The effects of systematic instruction on preschoolers' use of StayPlay-Talk with their peers with social delays. Journal of Early Intervention. https://doi.org/10.1177 $/ 1053815119900253$

Morgan, C. A., \& Wine, B. (2018). Evaluation of behavior skills training for teaching work skills to a student with autism spectrum disorder. Education \& Treatment of Children, 41(2), 223-232. https://doi.org/10.1007/s10803-017-3455-z.
Patry, M. B., \& Horn, E. (2020). Supporting the play of preschoolers with autism through peer-mediated interventions. Young Exceptional Children, 23(1), 3-14. https://doi. org/10.1177/1096250618782179.

Sawyer, M. R., Andzik, N. R., Kranak, M. P., Willke, C. P., Curiel, E. S. L., Hensley, L. E., \& Neef, N. A. (2017). Improving pre-service teachers' performance skills through behavioral skills training. Behavior Analysis in Practice, 10(3), 296-300. https://doi.org/10.1007/s40617-017-0198-4.

Simpson, L. A., \& Bui, Y. (2016). Effects of a peer-mediated intervention on social interactions of students with lowfunctioning autism and perceptions of typical peers. Education \& Training in Autism \& Developmental Disabilities, 51(2), 162-178. https://doi.org/10.1177 $/ 0271121419839136$.

Singh, B. D., Moore, D. W., Furlonger, B. E., Anderson, A., Busacca, M. L., \& English, D. L. (2017). Teaching reading comprehension skills to a child with autism using behaviour skills training. Journal of Autism \& Developmental Disorders, 47, 3049-3058. https://doi.org/10.1007/s10803017-3229-7.

Strain, P. S., Shores, R. E., \& Timm, M. A. (1977). Effects of peer social initiations on the behavior of withdrawn preschool children. Journal of Applied Behavior Analysis, 10, 289298. https://doi.org/10.1901/jaba.1977.10-289.

Tarasenko, M. A., Miltenberger, R. G., Brower-Breitwieser, C., \& Bosch, A. (2010). Evaluation of peer training for teaching abduction prevention skills. Child \& Family Behavior Therapy, 32(3), 219-230. https://doi.org/10.1080 /07317107.2010.500518.

Zhang, J., \& Wheeler, J. J. (2011). A meta-analysis of peermediated interventions for young children with autism spectrum disorders. Education \& Training in Autism \& Developmental Disabilities, 46(1), 62-77. https://doi. org/10.1007/s40489-014-0014-9. 